NORDITA-2004-17 HE

SUNY-NTG-04/01

\title{
Supersymmetric Quenching of the Toda Lattice Equation
}

\author{
K. Splittorff ${ }^{1}$ and J.J.M. Verbaarschot ${ }^{2}$ \\ 1 Nordita, Blegdamsvej 17, DK-2100, Copenhagen Ø, Denmark \\ ${ }^{2}$ Department of Physics and Astronomy, SUNY, Stony Brook, New York 11794, USA \\ email:split@alf.nbi.dk and verbaarschot@tonic.physics.sunysb.edu
}

(March 19, 2022)

The average of the ratio of powers of the spectral determinants of the Dirac operator in the $\epsilon$-regime of QCD is shown to satisfy a Toda lattice equation. The quenched limit of this Toda lattice equation is obtained using the supersymmetric method. This super symmetric approach is then shown to be equivalent to taking the replica limit of the Toda lattice equation. Among other, the factorization of the microscopic spectral correlation functions of the QCD Dirac operator into fermionic and bosonic partition functions follows naturally from both approaches. While the replica approach relies on an analytic continuation in the number of flavors no such assumptions are made in the present approach where the numbers of flavors in the Toda lattice equation are strictly integer. 


\section{INTRODUCTION}

A quenched theory is one in which a determinant has been removed from weight in the partition function. Removing a determinant from the partition function does not necessarily lead to an unphysical theory. The determinant can act as a source term for Green's functions, and, once a derivative of the partition function has been taken, this source is removed. Two widely used analytical tools to remove determinants are the replica trick and the supersymmetric method. For the replica trick, one introduces $n$ identical copies of the determinant, performs the appropriate derivatives, and finally takes the limit $n \rightarrow 0$. For the supersymmetric method, a ratio of two determinants with different values of the sources is introduced in the partition function. Derivatives are performed with respect to the sources and the determinants are removed by setting the sources equal. This paper deals with the equivalence of these two approaches.

Originally, both the replica trick [1] and the supersymmetric method [2] where invented for the study of disordered systems. Applications of the replica trick soon proved to be delicate, see eg. [3], the trouble being in the choice of the dominant saddle points and the analytic continuation in the replica index [4]. The supersymmetric method [2] does not rely on such analytic continuation, and never faces this problem. At the perturbative level, one only finds a polynomial dependence on the number of replicas and both approaches yield identical results (see for example [4-6]). The replica trick has made its mark in diverse areas of theoretical physics. Some examples are the proof of the linked cluster theorem (see [7]) and the study of vacua in supersymmetric gauge theories [8].

In disordered systems, which also includes the Dirac operator in a gauge field distributed according to the YangMills action, the standard approach both for the replica trick and the supersymmetric method is to rewrite the bosonic and/or fermionic integrals in terms of a non-linear $\sigma$-model. Such a $\sigma$-model describes the Goldstone modes associated with the spontaneous breaking of the global symmetries of the original model. Both its symmetry breaking term and its kinetic term are determined by the pattern of global symmetry breaking and Lorentz invariance. In the theory of disordered systems, these Goldstone modes are known as diffusons whereas in QCD they are known as the pseudoscalar mesons (for reviews see $[2,9]$ ). For QCD, the $\sigma$-model is known as a chiral Lagrangian and has been studied extensively in relation to the low-energy properties of the strong interactions [10].

In a parameter domain where the kinetic term is important, in most cases, only a perturbative treatment of the theory is feasible. However, in a domain where the kinetic term can be ignored, which is know as the ergodic domain or the $\epsilon$ domain, the $\sigma$-model becomes zero dimensional and can be solved exactly in many cases. The zero dimensional $\sigma$-models are equivalent to random matrix theories [2,11-14]. Using this connection, the problems with the replica trick where shown to be of a more serious nature [15]. This lead to the general consensus that the replica trick was only reliable for perturbative calculations and could generally not be used to obtain exact nonperturbative results. A first hint that nonperturbative results could be obtained from the replica trick came from $[16,17]$ where the oscillatory factors in the asymptotic expansion of the two-point correlations function were reproduced. However, only in cases where the perturbative expansion terminates [18], exact analytical results could be obtained, and, as was pointed out in [18], a systematic and exact replica method remained illusive in these works. Recently, replica methods were introduced that went beyond these limitations [19-22]. Most significantly, exact analytical results were obtained in cases where other methods have failed. While the formulation of the replica trick introduced in [20] has been shown to be widely applicable, it still relies on an assumed analytical continuation in the replica index. The central purpose of this paper is to justify this analytical continuation.

The new development which allowed for an exact analytic evaluation of the spectral correlation functions is the link between zero dimensional $\sigma$-models and an exactly solvable system know as the Toda lattice. This system is one dimensional and consists of massive particles interacting through an exponential potential [23]. The Hamilton equations of motion for the position of the masses as a function of time are known as the Toda lattice equations. The system is integrable, and its solution for appropriate boundary conditions is given by the partition function of a zero dimensional $\sigma$-model.

Previously, we have shown $[20,21]$ that the replica limit of the Toda lattice equation not only gives the exact analytic results but also explains the factorization of the universal microscopic correlation functions into a product of a bosonic and a fermionic partition function. Though this factorization suggests a direct relationship with the supersymmetric generating functionals, it has remained a miracle why the final result factorizes within the supersymmetric method. In this paper we show this factorization based on explicit expressions for the ratio of different powers of spectral determinants [24]. The key observation is that this family of partition functions also satisfies Toda lattice equations. Quenching these Toda equations as in the supersymmetric method, that is setting bosonic and fermionic masses equal, automatically reveals the factorization of correlation functions. As stressed above, the replica limit of the Toda lattice equation relies on an analytic continuation in the number of flavors from integer values to real values. Although the replica limit of the Toda lattice equation, in cases that have been studied up to now, has given the correct analytic result, no definite arguments have been given that the usual problems of the replica limit do not manifest themselves 
in this approach. As we will show in this paper the replica limit of the Toda lattice equation is equivalent to quenching the Toda lattice equation of a family of supersymmetric partition functions with a strictly integer number of bosonic and fermionic flavors. This result directly justifies the interpretation of the partition function with zero flavors in the usual Toda lattice equation as the supersymmetric partition function.

Throughout this paper we consider the zero dimensional limit of the $\sigma$-model relevant for QCD. In the random matrix formulation this corresponds to the chiral unitary ensemble (chUE). We expect that similar results can be obtained for the unitary ensemble.

This paper is organized as follows. First we describe the replica method and the supersymmetric method. Then, in section III, we discuss Toda lattice equations relevant for the one-point function and the two-point function. In section IV the quenching of the Toda lattice equations is discussed. This automatically yields the quenched correlation functions in the factorized form. The equivalence of the replica method and the supersymmetric approach is discussed in section V. Finally, in section VI, we summarize our results and comment on possible extensions of our work. In two appendices we derive the Toda lattice equations for the supersymmetric partition functions.

\section{DEFINITIONS OF REPLICA AND SUPERSYMMETRIC QUENCHING}

In this section we set our notation and introduce the supersymmetric method and the replica method. We illustrate the general definitions with generating functions for the microscopic limit of the one-point functions of the chUE.

The essential ingredient in the partition functions are the fermionic and bosonic determinants which will be written explicitly whereas all other parts of the weight are included in the average denoted by $\langle\ldots\rangle$. The fermionic replicated partition function is defined by

$$
Z_{N_{f}}(x)=\left\langle\operatorname{det}^{N_{f}}(D+x)\right\rangle,
$$

where $D$ is an operator, the mass $x$ is a number, and, as just mentioned, $\langle\ldots\rangle$ denotes the average for the specific theory (note that $\partial_{x}\langle 1\rangle=0$ ). Analogously, the bosonic replicated partition function is given by

$$
Z_{-N_{b}}(y)=\left\langle\operatorname{det}^{-N_{b}}(D+y)\right\rangle .
$$

The replica trick for removing the determinants is to take the limit $N_{f} \rightarrow 0$ or $N_{b} \rightarrow 0$.

The supersymmetric partition function is defined by the average ratio of powers of the spectral determinants

$$
Z_{N_{f},-N_{b}}(x \mid y)=\left\langle\operatorname{det}^{N_{f}}(D+x) \operatorname{det}^{-N_{b}}(D+y)\right\rangle
$$

which can be removed by taking the limit $y \rightarrow x$. For example,

$$
\lim _{y \rightarrow x} Z_{4,-2}(x \mid y)=Z_{2}(x) .
$$

Obviously, it is not particularly interesting to introduce and remove determinants unless we differentiate with respect to the masses first. The average resolvent of the operator $D$ is defined as

$$
G(x) \equiv\left\langle\operatorname{Tr} \frac{1}{D+x}\right\rangle .
$$

In the fermionic replica limit it is given by

$$
G(x)=\lim _{N_{f} \rightarrow 0} \frac{1}{N_{f}} \partial_{x} \log Z_{N_{f}}(x),
$$

in the bosonic replica limit we have that

$$
G(x)=\lim _{N_{b} \rightarrow 0} \frac{1}{-N_{b}} \partial_{x} \log Z_{-N_{b}}(x),
$$

and the supersymmetric method is based on the identity 


$$
G(x)=\lim _{y \rightarrow x} \partial_{x} \log Z_{1,-1}(x \mid y) .
$$

While the average in eq. (1) appears to be well defined for any real value of $N_{f}$ it is often not possible to evaluate the average unless $N_{f}$ is an integer. For this reason, taking the limit $N_{f} \rightarrow 0$ involves an analytic continuation in $N_{f}$.

In this paper we focus on the topological trivial sector of the QCD partition function which is the average of a fermionic determinant

$$
\mathcal{Z}_{N_{f}}\left(\left\{m_{f}\right\}\right)=\int[\mathrm{d} A]_{0}\left[\prod_{f=1}^{N_{f}} \operatorname{det}\left(i \gamma_{\mu} D_{\mu}-m_{f}\right)\right] e^{-S_{\mathrm{YM}}(A)}
$$

over gauge field configurations with trivial topology weighted by the Yang-Mills action $S_{\mathrm{YM}}$. The quark masses $\left\{m_{f}\right\} \equiv m_{1}, \ldots, m_{N_{f}}$ may take different or identical values. In particular, we are interested in spectral correlation functions of the Dirac operator $\gamma_{\mu} D_{\mu}$ in the phase where chiral symmetry is spontaneously broken. The order parameter for the spontaneous breakdown of chiral symmetry is the chiral condensate denoted by $\langle\bar{\psi} \psi\rangle$. At low energies, the effective degrees of freedom are the Goldstone modes associated with this spontaneously broken symmetry, and the effective theory is a $\sigma$-model [25]. The zero dimensional limit of this $\sigma$-model allows for an analytical evaluation of the correlation functions on microscopic scales. The microscopic scale is set by the inverse of $V\langle\bar{\psi} \psi\rangle$, where $V$ is volume of the system. The domain in which the zero momentum modes of this effective theory decouple from the nonzero modes is known as the $\epsilon$-regime of QCD [10]. In the topologically trivial sector, $\nu=0$, the fermionic partition functions are given by the unitary, and hence compact, integral [26-28]

$$
Z_{N_{f}}\left(\left\{x_{f}\right\}\right)=\int_{U \in U\left(N_{f}\right)} d U e^{\frac{1}{2} \operatorname{Tr}\left[M^{\dagger} U+M U^{\dagger}\right]},
$$

where $M \equiv \operatorname{diag}\left(x_{1}, \cdots, x_{N_{f}}\right) \equiv \operatorname{diag}\left(\langle\bar{\psi} \psi\rangle V m_{1}, \cdots,\langle\bar{\psi} \psi\rangle V m_{N_{f}}\right)$ is the rescaled mass matrix. The bosonic partition functions are given by a non-compact integral over positive definite matrices [13,29]

$$
Z_{-N_{b}}\left(\left\{y_{b}\right\}\right)=\int_{Q \in G l\left(N_{b}\right) / U\left(N_{b}\right)} d Q e^{-\frac{1}{2} \operatorname{Tr}\left[M^{\dagger} Q+M Q^{-1}\right]},
$$

where the dependence on the bosonic quark masses, the chiral condensate, and the volume is through the products $y_{b} \equiv m_{b}\langle\bar{\psi} \psi\rangle V$ appearing in the rescaled quark mass matrix $M \equiv \operatorname{diag}\left(y_{1}, \cdots, y_{N_{b}}\right)$. The supersymmetric partition function of QCD in the $\epsilon$-regime is given by an integral over a supergroup $[12,13]$

$$
Z_{N_{f},-N_{b}}\left(\left\{x_{f}\right\} \mid\left\{y_{b}\right\}\right)=\int_{Q \in \hat{G} l\left(N_{f} \mid N_{b}\right)} d Q e^{\frac{1}{2} \operatorname{Str}\left[M^{\dagger} Q+M Q^{-1}\right]}
$$

where $M=\operatorname{diag}\left(x_{1}, \cdots, x_{N_{f}}, y_{1}, \cdots, y_{N_{b}}\right)$ is the rescaled mass matrix. The boson-boson block of $\hat{G} l\left(N_{f} \mid N_{b}\right)$ is $G l\left(N_{b}\right) / U\left(N_{b}\right)$ and the fermion-fermion block of $\hat{G l}\left(N_{f} \mid N_{b}\right)$ is $U\left(N_{f}\right)$. All three integrals are fixed uniquely by the symmetries.

Much has been learned about these partition functions over the past few years. Explicit expressions in terms of determinants of Bessel functions are known in all cases [13,20,24,27,29,30,32-39]. For $Z_{N_{f}}$ and $Z_{-N_{b}}$ the expressions have been derived directly from the group integrals (10) and (11) while the derivation of the general expression for $Z_{N_{f},-N_{b}}[24,34-36]$ goes through the random matrix representation. For all the partition functions $N_{f}$ and $N_{b}$ must take integer values and the analytic continuation in $N_{f}$ and $N_{b}$ is not uniquely defined.

As mentioned in the introduction, the latest developments include a link between such matrix integrals and an exactly solvable one dimensional system known as the Toda lattice $[32,33,40]$. The Hamiltonian equation of motion of the Toda lattice is known as the Toda lattice equation. The remarkable link is that the QCD partition functions also obey such Toda lattice equation. This equation connects partition functions with different numbers of flavors, much in the same way as recursion relations for orthogonal polynomials connect polynomials with different indices. It turns out that the Toda lattice equation for fermionic flavors is also valid for bosonic flavors. Both in the bosonic case and the fermionic case the Toda lattice equation terminates for zero flavors. Based on the correct analytical result for the replica limit of these hierarchies, it was conjectured in [20] that both hierarchies are connected by the supersymmetric partition function. This assertion is proved in this paper starting from the observation that the supersymmetric partition functions also obey a Toda lattice equation. 


\section{THE TODA LATTICE EQUATIONS FOR THE SUPERSYMMETRIC PARTITION FUNCTION}

The Toda lattice equations considered in the literature (see for instance [40-42]) connect partition functions with a different number of fermionic flavors or a different number of bosonic flavors. That is, the Toda lattice equations deal with semi-infinite hierarchies with either positive or negative index. In [20,21] it was found that these two semi-infinite hierarchies are connected. The connection was realized as the replica limit of the index in either the positive or the negative semi-infinite hierarchy. Here, we consider Toda lattice equations for the partition functions with both fermions and bosons, i.e. super-symmetric partition functions. Referring to the graded symmetry of the supersymmetric partition functions we call these graded Toda lattice equations. In the next section we will derive the quenched resolvents from these graded Toda lattice equations.

\section{A. A graded Toda Lattice Equation Affecting One Flavor}

Starting from the expression for the partition function (12) with $N_{f}$ fermionic flavors and $N_{b}$ bosonic flavors $[34-36,20]^{*}$

$$
Z_{N_{f},-N_{b}}\left(\left\{x_{f}\right\} \mid\left\{y_{b}\right\}\right)=\frac{\operatorname{det}\left[z_{i}^{j-1} \mathcal{J}_{j-1}\left(z_{i}\right)\right]_{i, j=1, . ., N_{f}+N_{b}}}{\prod_{j>i=1}^{N_{f}}\left(x_{j}^{2}-x_{i}^{2}\right) \prod_{j>i=1}^{N_{b}}\left(y_{j}^{2}-y_{i}^{2}\right)}
$$

where $z_{i}=x_{i}$ for $i=1, \ldots, N_{f}, z_{N_{f}+i}=y_{i}$ for $i=1, \ldots, N_{b}, \mathcal{J}_{j-1}\left(z_{i}\right) \equiv I_{j-1}\left(x_{i}\right)$ for $i=1, \ldots, N_{f}$, and $\mathcal{J}_{j-1}\left(z_{N_{f}+i}\right) \equiv(-1)^{j-1} K_{j-1}\left(y_{i}\right)$ for $i=1, \ldots, N_{b}$, we prove in Appendix A that the partition function (12) satisfies the graded Toda lattice relation

$$
\delta_{N_{f}+N_{b}} \delta_{x_{i}} \log Z_{N_{f},-N_{b}}\left(\left\{x_{f}\right\} \mid\left\{y_{b}\right\}\right)=2 n x_{i}^{2} \frac{Z_{N_{f}+1,-N_{b}}\left(x_{i},\left\{x_{f}\right\} \mid\left\{y_{b}\right\}\right) Z_{N_{f}-1,-N_{b}}\left(\left\{x_{f}\right\}_{f \neq i} \mid\left\{y_{b}\right\}\right)}{\left[Z_{N_{f},-N_{b}}\left(\left\{x_{k}\right\} \mid\left\{y_{b}\right\}\right)\right]^{2}}
$$

Here, $n$ is the number of fermionic flavors with mass $x_{i}$ in $Z_{N_{f},-N_{b}}\left(\left\{x_{f}\right\} \mid\left\{y_{b}\right\}\right)$. The derivation $\delta_{x}$ is defined by

$$
\delta_{x} \equiv x \partial_{x}
$$

and

$$
\delta_{N_{f}+N_{b}} \equiv \sum_{f=1}^{N_{f}} \delta_{x_{f}}+\sum_{b=1}^{N_{b}} \delta_{y_{b}}
$$

For degenerate masses the sum only extends over the different masses.

This is the generalization of the Toda lattice equation for fermions obtained in [42], to include both fermions and bosons. Below, in section IV A, we quench this graded Toda lattice equation as in the supersymmetric method and show that the factorization of the quenched resolvent follows naturally.

\section{B. A graded Toda Lattice Equation Affecting Two Flavors}

The key ingredient in understanding the factorization property [21] of the two-point correlation function within the supersymmetric approach is a graded Toda lattice equation where two indices are raised and lowered simultaneously. In [21] it was shown that for $m$ degenerate fermionic flavors with mass $x_{1}$ and $n$ degenerate fermionic flavors with mass $x_{2}$, the partition function (10) satisfies the Toda lattice equation ( $m$ and $n$ are positive integers)

\footnotetext{
*Notation: The vertical bar in the argument of $Z$ separates the masses $\left\{x_{f}\right\} \equiv x_{1}, \ldots, x_{N_{f}}$ of the fermionic quarks from the masses $\left\{y_{b}\right\} \equiv y_{1}, \ldots, y_{N_{b}}$ of the bosonic quarks. For degenerate masses we either repeat the mass in the argument of $Z$ according to the degeneracy or put an additional subscript on $Z$ indicating the degeneracy, eg. $Z_{3,1,-2}\left(x_{1}, x_{2} \mid y\right) \equiv Z_{4,-2}\left(x_{1}, x_{1}, x_{1}, x_{2} \mid y, y\right)$. For bosonic masses, the subscritpt of $Z$ that denotes the number of flavors will contain and explicit minus sign. If all flavors are of the same kind the vertical bar will be omitted.
} 


$$
\delta_{x_{1}} \delta_{x_{2}} \log Z_{m, n}\left(x_{1}, x_{2}\right)=4 m n x_{1}^{2} x_{2}^{2} \frac{Z_{m+1, n+1}\left(x_{1}, x_{2}\right) Z_{m-1, n-1}\left(x_{1}, x_{2}\right)}{\left[Z_{m, n}\left(x_{1}, x_{2}\right)\right]^{2}}
$$

Using the explicit expressions for the partition function given in (13) we have shown that

$$
\delta_{x_{1}} \delta_{x_{2}} \log Z_{2,-2}\left(x_{1}, x_{2} \mid y_{1}, y_{2}\right)=4 x_{1}^{2} x_{2}^{2} \frac{Z_{4,-2}\left(x_{1}, x_{1}, x_{2}, x_{2} \mid y_{1}, y_{2}\right) Z_{-2}\left(y_{1}, y_{2}\right)}{\left.\left[Z_{2,-2}\left(x_{1}, x_{2} \mid y_{1}, y_{2}\right)\right)\right]^{2}}
$$

and a similar equation for other small numerical values of the number of flavors (see Appendix B).

We conjecture that these results can be generalized to any integer number of fermions, $N_{f}$, and any integer number of bosons, $N_{b}$ (further support for this conjecture will be given in Appendix B):

$$
\delta_{x_{i}} \delta_{x_{j}} \log Z_{N_{f},-N_{b}}\left(\left\{x_{f}\right\} \mid\left\{y_{b}\right\}\right)=4 n m x_{i}^{2} x_{j}^{2} \frac{Z_{N_{f}+2,-N_{b}}\left(x_{i}, x_{j},\left\{x_{f}\right\} \mid\left\{y_{b}\right\}\right) Z_{N_{f}-2,-N_{b}}\left(\left\{x_{f}\right\}_{f \neq i, j} \mid\left\{y_{b}\right\}\right)}{\left[Z_{N_{f},-N_{b}}\left(\left\{x_{f}\right\} \mid\left\{y_{b}\right\}\right)\right]^{2}},
$$

where $m$ and $n$ are the number of flavors with mass $x_{i}$ and $x_{j}$ in $Z_{N_{f},-N_{b}}\left(\left\{x_{f}\right\} \mid\left\{y_{b}\right\}\right)$, respectively. A similar equation holds for differentiation with respect to two bosonic masses. In that case the fermionic flavors are passive. Furthermore, we have shown for a number of values of $N_{f}$ and $N_{b}$ (see Appendix B) that a corresponding equation is valid when we differentiate with respect to one fermionic and one bosonic mass. Without proof we assert that the general result in this case is given by

$$
\delta_{x_{i}} \delta_{y_{j}} \log Z_{N_{f},-N_{b}}\left(\left\{x_{f}\right\} \mid\left\{y_{b}\right\}\right)=-4 n m x_{i}^{2} y_{j}^{2} \frac{Z_{N_{f}+1,-N_{b}+1}\left(x_{i},\left\{x_{f}\right\} \mid\left\{y_{b}\right\}_{b \neq j}\right) Z_{N_{f}-1,-N_{b}-1}\left(\left\{x_{f}\right\}_{f \neq i} \mid y_{j},\left\{y_{b}\right\}\right)}{\left[Z_{N_{f},-N_{b}}\left(\left\{x_{f}\right\} \mid\left\{y_{b}\right\}\right)\right]^{2}}
$$

where $m$ and $n$ are the number of flavors with mass $x_{i}$ and $y_{j}$ in $Z_{N_{f},-N_{b}}\left(\left\{x_{f}\right\} \mid\left\{y_{b}\right\}\right)$. These graded Toda lattice equations go beyond previous results [21] in two respects: 1) they have been extended to supersymmetric partition functions, and 2) the masses in these equations can be either degenerate or non degenerate.

\section{SUPERSYMMETRIC QUENCHING OF THE GRADED TODA LATTICE EQUATION}

In the supersymmetric approach the partially quenched or fully quenched correlation functions are obtained by differentiating before taking the limit of equal fermionic and bosonic masses. In this section we show how the graded Toda lattice equations of the previous section automatically give the quenched one-point and two-point functions. The results are in exact agreement with expressions obtained previously. The important new result of this section is that the factorization of correlation functions into products of partition functions follows naturally without assuming an analytical continuation in the number of flavors.

\section{A. The One Point Function}

In this section we derive the quenched spectral one point function by quenching the graded Toda lattice equation (14) using the supersymmetric method. For this it is sufficient to have one bosonic and one fermionic flavor, and the graded Toda lattice equation then reads

$$
\delta_{x}\left(\delta_{x}+\delta_{y}\right) \log Z_{1,-1}(x \mid y)=2 x^{2} \frac{Z_{2,-1}(x, x \mid y) Z_{-1}(y)}{\left[Z_{1,-1}(x \mid y)\right]^{2}} .
$$

the limit $y \rightarrow x$ of the l.h.s. of this equation is given by

$$
\begin{aligned}
\lim _{y \rightarrow x} \delta_{x}\left(\delta_{x}+\delta_{y}\right) \log Z_{1,-1}(x \mid y) & =\lim _{y \rightarrow x} \delta_{x}\left[x\left\langle\operatorname{Tr} \frac{1}{D+x}\right\rangle_{x, y}-y\left\langle\operatorname{Tr} \frac{1}{D+y}\right\rangle_{x, y}\right] \\
& =\delta_{x} x G(x) .
\end{aligned}
$$

Here, we have used the notation

$$
\langle\ldots\rangle_{x, y}=\left\langle\ldots \frac{\operatorname{det}(D+x)}{\operatorname{det}(D+y)}\right\rangle
$$


Taking also the limit $y \rightarrow x$ of the r.h.s. of (21) we find

$$
\delta_{x} x G(x)=2 x^{2} Z_{1}(x) Z_{-1}(x) .
$$

This result is in complete agreement with known results for the resolvent and the partition functions. For completeness we mention that they are given by

$$
G(x)=x\left(K_{0}(x) I_{0}(x)+K_{-1}(x) I_{1}(x)\right), \quad Z_{1}(x)=I_{0}(x), \quad Z_{-1}(x)=K_{0}(x) .
$$

Clearly, this is a rather cumbersome way of deriving the resolvent from $Z_{1,-1}$. Let us therefore again stress that the important new result here is that the factorization in eq. (24) is proved on the basis of the Toda lattice hierarchy for the supersymmetric partition functions. We also stress that this approach does not rely on an analytic continuation in the number of flavors.

Following the same strategy as above one can also obtain the partially quenched resolvents. As we seek to stress a matter of principle rather than reproducing existing results we instead turn to the two-point function.

\section{B. The Two-Point Function}

The two-point disconnected susceptibility contains two derivatives just as in the Toda lattice equation. This property simplifies the derivation of two point-functions from the Toda lattice equation for ratios of spectral determinants.

The fully quenched disconnected susceptibility in the chiral unitary ensemble with identical arguments is defined as

$$
\chi(x, x) \equiv-\lim _{y \rightarrow x} \partial_{x} \partial_{y} \log Z_{1,-1}(x \mid y) .
$$

It follows from (20) for $N_{f}=N_{b}=1$ at equal values of the arguments that

$$
\chi(x, x) \equiv-\lim _{y \rightarrow x} \partial_{x} \partial_{y} \log Z_{1,-1}(x \mid y)=4 x^{2} Z_{2}(x, x) Z_{-2}(x, x) .
$$

In order to get the quenched disconnected susceptibility for different values of the arguments, we start from the partition function describing two fermions and two bosons all with different masses. We now have a choice: we can differentiate with respect to two fermionic masses, with respect to two bosonic masses, or with respect to one fermionic mass and one bosonic mass, before pairing up the fermion and boson masses. If we choose the first option and use (18) we find

$$
\begin{aligned}
\chi\left(x_{1}, y_{1}\right) & \equiv-\lim _{y_{1} \rightarrow x_{1}, y_{2} \rightarrow x_{2}} \partial_{x_{1}} \partial_{x_{2}} \log Z_{2,-2}\left(x_{1}, x_{2} \mid y_{1}, y_{2}\right) \\
& =\lim _{y_{1} \rightarrow x_{1}, y_{2} \rightarrow x_{2}} 4 x_{1} x_{2} \frac{Z_{4,-2}\left(x_{1}, x_{1}, x_{2}, x_{2} \mid y_{1}, y_{2}\right) Z_{-2}\left(y_{1}, y_{2}\right)}{\left[Z_{2,-2}\left(x_{1}, x_{2} \mid y_{1}, y_{2}\right)\right]^{2}} \\
& =4 x_{1} x_{2} Z_{2}\left(x_{1}, x_{2}\right) Z_{-2}\left(x_{1}, x_{2}\right) .
\end{aligned}
$$

The derivation when differentiating with respect to the two bosonic masses is analogous and leads to exactly the same quenched correlation function. If we choose to differentiate with respect to one fermionic mass and one bosonic mass and use (20) for $N_{f}=N_{b}=2$,

$$
\partial_{x_{2}} \partial_{y_{1}} \log Z_{2,-2}\left(x_{1}, x_{2} \mid y_{1}, y_{2}\right)=-4 x_{2} y_{1} \frac{Z_{3,-1}\left(x_{1}, x_{2}, x_{2} \mid y_{2}\right) Z_{1,-3}\left(x_{1} \mid y_{1}, y_{1}, y_{2}\right)}{\left[Z_{2,-2}\left(x_{1}, x_{2} \mid y_{1}, y_{2}\right)\right]^{2}},
$$

(which we have also verified explicitly) we obtain

$$
\begin{aligned}
\chi\left(x_{1}, x_{2}\right) & \equiv-\lim _{y_{1} \rightarrow x_{1}, y_{2} \rightarrow x_{2}} \partial_{x_{2}} \partial_{y_{1}} \log Z_{2,-2}\left(x_{1}, x_{2} \mid y_{1}, y_{2}\right) \\
& =\lim _{y_{1} \rightarrow x_{1}, y_{2} \rightarrow x_{2}} 4 x_{2} y_{1} \frac{Z_{3,-1}\left(x_{1}, x_{2}, x_{2} \mid y_{2}\right) Z_{1,-3}\left(x_{1} \mid y_{1}, y_{1}, y_{2}\right)}{\left[Z_{2,-2}\left(x_{1}, x_{2} \mid y_{1}, y_{2}\right)\right]^{2}} \\
& =4 x_{1} x_{2} Z_{2}\left(x_{1}, x_{2}\right) Z_{-2}\left(x_{1}, x_{2}\right) .
\end{aligned}
$$

This result coincides with (28) and is in agreement with the result obtained from a supersymmetric calculation [43] and with the result obtained from the replica limit of the Toda lattice equation of the fermionic hierarchy [21]. Most importantly, it is clear that the factorization property of the quenched two-point function in the supersymmetric approach is a direct consequence of the structure in the graded Toda lattice equation. No assumptions on the analytic continuation in the flavor index have to be made in this case. 


\section{EQUIVALENCE OF REPLICA AND SUPERSYMMETRIC QUENCHING}

In this section we show that the replica limit of the Toda lattice equation necessarily gives the same answer as the supersymmetrically quenched Toda lattice equation. This directly justifies the central assumption of [20,21], namely that the bosonic and fermionic semi-infinite Toda lattice hierarchies are connected by the supersymmetric partition function.

\section{A. Equivalence for the one Point Function}

The QCD partition function (10) with $N_{f}$ mass degenerate fermionic quarks satisfies the Toda lattice equation [42]

$$
\delta_{x}^{2} \log Z_{N_{f}}(x)=2 N_{f} x^{2} \frac{Z_{N_{f}+1}(x) Z_{N_{f}-1}(x)}{\left[Z_{N_{f}}(x)\right]^{2}} .
$$

(Note that this is obtained from (14), satisfied by the supersymmetric partition function, setting $n=N_{f}$ and $N_{b}=0$.) In the replica approach we would like to take the $N_{f} \rightarrow 0$ limit of this equation. Using the representation (1) of the partition function, which depends continuously on $N_{f}$, we get [20]

$$
\begin{aligned}
\lim _{N_{f} \rightarrow 0} \frac{1}{N_{f}} \delta_{x}^{2} \log Z_{N_{f}}(x) & =\delta_{x} x G(x), \\
\lim _{N_{f} \rightarrow 0} 2 x^{2} \frac{Z_{N_{f}+1}(x) Z_{N_{f}-1}(x)}{\left[Z_{N_{f}}(x)\right]^{2}} & =2 x^{2} Z_{1}(x) Z_{-1}(x) .
\end{aligned}
$$

However, the Toda lattice equation (31) was established starting from (10), which is only well defined for positive integer values of $N_{f}$, and the boundary condition $Z_{N_{f}=0}(x)=1$. The Toda lattice equation (31) therefore is not necessarily valid for (1) when $N_{f}$ is non-integer. However, as we will now show, equating (32) to (33) is identical to quenching the Toda lattice equation using the supersymmetric method, since the supersymmetric partition function satisfies the Toda lattice equation. To this end we write (31) as

$$
\frac{1}{N_{f}} \delta_{x}^{2} \log Z_{N_{f}}(x)=2 x^{2} \frac{Z_{N_{f}+1}(x) Z_{N_{f}-1}(x)}{\left[Z_{N_{f}}(x)\right]^{2}}=\lim _{y \rightarrow x} 2 x^{2} \frac{Z_{N_{f}+2,-1}(x \mid y) Z_{N_{f},-1}(x \mid y)}{\left[Z_{N_{f}+1,-1}(x \mid y)\right]^{2}} .
$$

These equalities are valid for positive integer values of $N_{f}$. However, the r.h.s. of the second equality is also well defined for $N_{f}=0$. If we take this as the definition of the $N_{f} \rightarrow 0$ limit of the middle term of this equation we get $2 x^{2} Z_{1}(x) Z_{-1}(x)$ exactly as in $(33)$, but now without ambiguities in the interpretation of $Z_{-1}(x)$. Moreover, in appendix $A$ it is shown that the supersymmetric partition function entering the r.h.s satisfies the same Toda lattice equation

$$
\delta_{x}\left(\delta_{x}+\delta_{y}\right) \log Z_{N_{f}+1,-1}(x \mid y)=2\left(N_{f}+1\right) x^{2} \frac{Z_{N_{f}+2,-1}(x \mid y) Z_{N_{f},-1}(x \mid y)}{\left[Z_{N_{f}+1,-1}(x \mid y)\right]^{2}} .
$$

This equation is also well-defined for $N_{f}=0$ and in this case the limit $y \rightarrow x$ is (cf. (22))

$$
\lim _{y \rightarrow x} \delta_{x}\left(\delta_{x}+\delta_{y}\right) \log Z_{1,-1}(x \mid y)=\delta_{x} x G(x) .
$$

This is precisely what we obtained in (32) using the representation (1) of the fermionic partition function. This shows that the replica limit of the Toda lattice equation is necessarily identical to the quenched limit of the supersymmetric Toda lattice equation.

\section{B. Equivalence for the Two-Point Function}

We now consider the replica limit of the Toda lattice equation affecting two indices simultaneously. In [21] it was shown that for integer $n>0$

$$
\partial_{x_{1}} \partial_{x_{2}} \log \left[Z_{n, n}\left(x_{1}, x_{2}\right)\right]=4 n^{2} x_{1} x_{2} \frac{Z_{n+1, n+1}\left(x_{1}, x_{2}\right) Z_{n-1, n-1}\left(x_{1}, x_{2}\right)}{\left[Z_{n, n}\left(x_{1}, x_{2}\right)\right]^{2}}
$$


Making the analytic continuation in $n$ it was assumed [21] that the limit $n \rightarrow 0$ connects this semi-infinite hierarchy to the semi-infinite hierarchy for $Z_{-n,-n}$, viz.

$$
\lim _{n \rightarrow 0} \frac{1}{n^{2}} \partial_{x_{1}} \partial_{x_{2}} \log \left[Z_{n, n}\left(x_{1}, x_{2}\right)\right]=4 x_{1} x_{2} Z_{1,1}\left(x_{1}, x_{2}\right) Z_{-1,-1}\left(x_{1}, x_{2}\right) .
$$

While the exact agreement of the resulting quenched two-point correlation function with the known result [43] vindicates this replica limit, the underlying assumption again could not be proved.

We now prove the assumption for the two-point function using similar arguments as for the one-point function. Again we establish the troublesome connection between positive and negative integer values of $n$ by quenching determinants as in the supersymmetric method. The ratio of partition functions in the r.h.s. of (37) can be written as

$$
\frac{Z_{n+1, n+1}\left(x_{1}, x_{2}\right) Z_{n-1, n-1}\left(x_{1}, x_{2}\right)}{\left[Z_{n, n}\left(x_{1}, x_{2}\right)\right]^{2}}=\lim _{y_{1} \rightarrow x_{1}, y_{2} \rightarrow x_{2}} \frac{Z_{n+2, n+2,-1,-1}\left(x_{1}, x_{2} \mid y_{1}, y_{2}\right) Z_{n, n,-1,-1}\left(x_{1}, x_{2} \mid y_{1}, y_{2}\right)}{\left[Z_{n+1, n+1,-1,-1}\left(x_{1}, x_{2} \mid y_{1}, y_{2}\right)\right]^{2}} .
$$

As discussed in section IV B the ratio on the r.h.s. satisfies a Toda recursion relation even before taking the limit of degenerate masses

$$
4(n+1)^{2} x_{1} x_{2} \frac{Z_{n+2, n+2,-1,-1}\left(x_{1}, x_{2} \mid y_{1}, y_{2}\right) Z_{n, n,-1,-1}\left(x_{1}, x_{2} \mid y_{1}, y_{2}\right)}{\left[Z_{n+1, n+1,-1,-1}\left(x_{1}, x_{2} \mid y_{1}, y_{2}\right)\right]^{2}}=\partial_{x_{1}} \partial_{x_{2}} \log \left[Z_{n+1, n+1,-1,-1}\left(x_{1}, x_{2} \mid y_{1}, y_{2}\right)\right]
$$

In this relation we can safely replace $n$ by zero so that

$$
\lim _{n \rightarrow 0} \frac{1}{n^{2}} \partial_{x_{1}} \partial_{x_{2}} \log \left[Z_{n, n}\left(x_{1}, x_{2}\right)\right]=\lim _{y_{1} \rightarrow x_{1}, y_{2} \rightarrow x_{2}} \partial_{x_{1}} \partial_{x_{2}} \log \left[Z_{1,1,-1,-1}\left(x_{1}, x_{2} \mid y_{1}, y_{2}\right)\right] .
$$

This shows that the replica limit of the Toda lattice equation necessarily gives the same answer as the supersymmetrically quenched Toda lattice equation, thus justifying the analytic continuation in $n$.

\section{CONCLUSION}

The QCD partition function in the $\epsilon$ regime is equivalent to the microscopic limit of a chiral random matrix theory with the same global symmetries. This equivalence holds for any number of bosonic and fermionic quarks, and each partition function in this family can be expressed as a determinant of Bessel functions. In this paper we have shown that this family of partition functions satisfies a Toda lattice equation which we have called the graded Toda lattice equation. Based on this result we have shown that the semi-infinite hierarchy of bosonic or fermionic partition functions can be continued to the limit of zero flavors which is given by the supersymmetric partition function. This was the central assumption when taking the replica limit of the Toda lattice equation. By proving this assumption we have thus shown the equivalence of the replica limit of the Toda lattice equation and the supersymmetric method. Quenching the partition functions in the graded Toda lattice equation shows that the resolvent of the supersymmetric partition function factorizes into the product of a bosonic and a fermionic partition function.

In its simplest form, the graded Toda lattice equation relates derivatives of the supersymmetric partition function to partition functions with one more flavor or with one flavor less. This case is relevant for the analysis of the onepoint function and has been proved in all its generality. For the analysis of the two-point function we need a graded Toda lattice equation in which derivatives of the partition function are related to partition functions with two more flavors or two less flavors. In addition to the cases that were required for our analysis, this Toda lattice equation was proved for several other cases with a fixed number of flavors. Based on these results and known results for exclusively fermionic flavors, we have conjectured that this equation can be generalized to an arbitrary number of bosonic and fermionic flavors.

It is not yet clear whether variants of the Toda lattice equation are suitable for the investigation of the replica limit of real symmetric $\left(\beta_{D}=1\right)$ and quaternion real $\left(\beta_{D}=4\right)$ random matrix ensembles. However, our work can be extended in several other directions. First, our results can be extended in a straightforward way to partition functions with a nonzero topological charge. The reason that we considered only zero topological charge in this paper is that it somewhat simplifies our notation. Second, in the formulation of Kanzieper, the replica limit is taken of the Painlevé equation rather than the Toda lattice equations. It would be very interesting to understand if the supersymmetric partition functions can also be incorporated in this framework. Third, the spectral density of the quenched Dirac operator at non-zero chemical potential in the limit of weak non-hermiticity was obtained from the replica limit of a Toda lattice equation. We expect that the supersymmetric generating functional at non-zero chemical potential will 
satisfy a similar Toda lattice equation so that the replica limit can also be justified in this case. Fourth, the two-point function of the unitary ensemble can also be obtained from the replica limit of a Toda lattice equation. Also in this case we believe that a generalization of the Toda lattice equation to supersymmetric partition functions is possible. Finally, we have no doubt that a similar arguments can be applied to QCD in 3 dimensions.

Acknowledgments: We wish to thank Eugene Kanzieper for useful correspondence on Painlevé equations and Poul Henrik Damgaard for discussions of the replica trick. This work was supported in part by U.S. DOE Grant No. DE-FG-88ER40388.

\section{Appendix A. THE GRADED TODA LATTICE EQUATION WHERE ONE INDEX IS AFFECTED}

In this appendix we prove the graded Toda lattice equation (14) relevant for the partially quenched one point functions, that is the relation where one flavor is added and subtracted. We start by slightly rewriting the general expression for the supersymmetric partition function in (13) [34-36,20]

$$
Z_{N_{f},-N_{b}}\left(\left\{x_{f}\right\} \mid\left\{y_{b}\right\}\right)=\frac{\operatorname{det}\left[\delta_{z_{i}}^{j-1} \mathcal{J}\left(z_{i}\right)\right]_{i, j=1, \ldots, N_{f}+N_{b}}}{\prod_{j>i=1}^{N_{f}}\left(x_{j}^{2}-x_{i}^{2}\right) \prod_{j>i=1}^{N_{b}}\left(y_{j}^{2}-y_{i}^{2}\right)} .
$$

Here, $z_{i}=x_{i}$ for $i=1, \ldots, N_{f}$, and $z_{N_{f}+i}=y_{i}$ for $i=1, \ldots, N_{b}$. Furthermore, the symbol $\mathcal{J}\left(z_{i}\right) \equiv I_{0}\left(x_{i}\right)$ for $i=1, \ldots, N_{f}$ and $\mathcal{J}\left(z_{N_{f}+i}\right) \equiv K_{0}\left(y_{i}\right)$ for $i=1, \ldots, N_{b}$. In order to keep track of the indices let us consider the last two fermionic flavors as mass degenerate, that is $x_{N_{f}}=x_{N_{f}+1}$. In this case the partition function is equal to

$$
Z_{N_{f}+1,-N_{b}}\left(x_{N_{f}},\left\{x_{f}\right\}_{f=1, \ldots, N_{f}} \mid\left\{y_{b}\right\}\right)=\frac{\operatorname{det}\left[A_{N_{f}+1,-N_{b}}\left(x_{N_{f}},\left\{x_{f}\right\}_{f=1, . ., N_{f}} \mid\left\{y_{b}\right\}\right)\right]}{\prod_{j>i=1}^{N_{f}}\left(x_{j}^{2}-x_{i}^{2}\right) \prod_{i=1}^{N_{f}-1}\left(x_{N_{f}}^{2}-x_{i}^{2}\right) 2 x_{N_{f}} \prod_{j>i=1}^{N_{b}}\left(y_{j}^{2}-y_{i}^{2}\right)},
$$

where we have introduced the $\left(N_{f}+1+N_{b}\right) \times\left(N_{f}+1+N_{b}\right)$ matrix

$$
A_{N_{f}+1,-N_{b}}\left(x_{N_{f}},\left\{x_{f}\right\}_{f=1, . ., N_{f}} \mid\left\{y_{b}\right\}\right) \equiv\left(\begin{array}{cccc}
I_{0}\left(x_{1}\right) & \delta_{x_{1}} I_{0}\left(x_{1}\right) & \cdots & \delta_{x_{1}}^{N_{f}+N_{b}} I_{0}\left(x_{1}\right) \\
\vdots & & & \vdots \\
I_{0}\left(x_{N_{f}}\right) & \cdots & \delta_{x_{N_{f}}}^{N_{f}+N_{b}} I_{0}\left(x_{N_{f}}\right) \\
\partial_{x_{N_{f}}} I_{0}\left(x_{N_{f}}\right) & \cdots & \partial_{x_{N_{f}}} \delta_{x_{f}}^{N_{f}+N_{b}} I_{0}\left(x_{N_{f}}\right) \\
K_{0}\left(y_{1}\right) & \delta_{y_{1}} K_{0}\left(y_{1}\right) & \cdots & \delta_{y_{1}}^{N_{f}+N_{b}} K_{0}\left(y_{1}\right) \\
\vdots & & & \vdots \\
K_{0}\left(y_{N_{b}}\right) & & \cdots & \delta_{y_{N_{b}}}^{N_{f}+N_{b}} K_{0}\left(y_{N_{b}}\right)
\end{array}\right) .
$$

The proof of the graded Toda lattice equation follows from the Sylvester identity [44] valid for the determinant of any matrix

$$
C_{i, j} C_{p, q}-C_{i, q} C_{p, j}=\operatorname{det}(A) C_{i, j ; p, q},
$$

where $C_{i, j}$ is the cofactor of matrix element $i j$

$$
C_{i, j} \equiv \frac{\partial \operatorname{det}(A)}{\partial A_{i j}}
$$

and $C_{i, j ; p, q}$ is the double cofactor of matrix elements $i j$ and $p q$

$$
C_{i, j ; p, q} \equiv \frac{\partial^{2} \operatorname{det}(A)}{\partial A_{i j} \partial A_{p q}}
$$

Setting $i=N_{f}+1, j=N_{f}+1+N_{b}, p=N_{f}$, and $q=N_{f}+N_{b}$ we have

$$
C_{N_{f}+1, N_{f}+1+N_{b}} C_{N_{f}, N_{f}+N_{b}}-C_{N_{f}+1, N_{f}+N_{b}} C_{N_{f}, N_{f}+1+N_{b}}=\operatorname{det}\left(A_{N_{f}+1,-N_{b}}\right) C_{N_{f}+1, N_{f}+1+N_{b} ; N_{f}, N_{f}+N_{b}} .
$$


From the structure (44) of the matrix $A_{N_{f}+1, N_{b}}$ it follows that $\left(\delta_{N_{f}+N_{b}}\right.$ is defined in (16))

$$
\begin{aligned}
C_{N_{f}+1, N_{f}+1+N_{b}} & =\operatorname{det} A_{N_{f},-N_{b}}, \\
C_{N_{f}, N_{f}+N_{b}} & =\frac{1}{x_{N_{f}}} \delta_{x_{N_{f}}} \delta_{N_{f}+N_{b}} \operatorname{det} A_{N_{f},-N_{b}}, \\
C_{N_{f}+1, N_{f}+N_{b}} & =\delta_{N_{f}+N_{b}} \operatorname{det} A_{N_{f},-N_{b}}, \\
C_{N_{f}, N_{f}+1+N_{b}} & =\frac{1}{x_{N_{f}}} \delta_{x_{N_{f}}} \operatorname{det} A_{N_{f},-N_{b}}, \\
C_{N_{f}+1, N_{f}+1+N_{b} ; N_{f}, N_{f}+N_{b}} & =\operatorname{det} A_{N_{f}-1,-N_{b}} .
\end{aligned}
$$

For completeness we write out

$$
A_{N_{f},-N_{b}}\left(\left\{x_{f}\right\} \mid\left\{y_{b}\right\}\right) \equiv\left(\begin{array}{cccc}
I_{0}\left(x_{1}\right) & \delta_{x_{1}} I_{0}\left(x_{1}\right) & \cdots & \delta_{x_{1}}^{N_{f}-1+N_{b}} I_{0}\left(x_{1}\right) \\
\vdots & & & \vdots \\
I_{0}\left(x_{N_{f}}\right) & & \cdots & \delta_{x_{N_{f}}}^{N_{f}-1+N_{b}} I_{0}\left(x_{N_{f}}\right) \\
K_{0}\left(y_{1}\right) & \delta_{y_{1}} K_{0}\left(y_{1}\right) & \cdots & \delta_{y_{1}}^{N_{f}-1+N_{b}} K_{0}\left(y_{1}\right) \\
\vdots & & & \vdots \\
K_{0}\left(y_{N_{b}}\right) & & \cdots & \delta_{y_{N_{b}}}^{N_{f}-1+N_{b}} K_{0}\left(y_{N_{b}}\right)
\end{array}\right) .
$$

Inserting this in (48) we get

$$
\left[\operatorname{det} A_{N_{f},-N_{b}}\right]^{2} \delta_{x_{N_{f}}} \delta_{N_{f}+N_{b}} \log \left[\operatorname{det} A_{N_{f},-N_{b}}\right]=x_{N_{f}} \operatorname{det} A_{N_{f}+1,-N_{b}} \operatorname{det} A_{N_{f}-1,-N_{b}} .
$$

Finally, using the relation (43) between the partition functions and the matrices $A$ we obtain the desired graded Toda lattice equation

$$
\delta_{N_{f}+N_{b}} \delta_{x_{i}} \log Z_{N_{f},-N_{b}}\left(\left\{x_{f}\right\} \mid\left\{y_{b}\right\}\right)=2 x_{i}^{2} \frac{Z_{N_{f}+1,-N_{b}}\left(x_{i},\left\{x_{f}\right\} \mid\left\{y_{b}\right\}\right) Z_{N_{f}-1,-N_{b}}\left(\left\{x_{f}\right\}_{f \neq i} \mid\left\{y_{b}\right\}\right)}{\left[Z_{N_{f},-N_{b}}\left(\left\{x_{k}\right\} \mid\left\{y_{b}\right\}\right)\right]^{2}} .
$$

Note that the Vandermonde determinants, $\Delta\left(\left\{z_{i}^{2}\right\}\right)$, in the squared masses do not contribute to the left hand side of the Toda lattice equation since $\delta_{N_{f}+N_{b}} \log \left[\Delta\left(\left\{z_{i}^{2}\right\}\right)\right]$ is a number.

In the case where $n$ flavors have mass $x_{N_{f}}$ the proof is similar. The partition function is now given by

$$
Z_{n, N_{f}-1,-N_{b}}\left(x_{N_{f}},\left\{x_{f}\right\}_{f \neq N_{f}} \mid\left\{y_{b}\right\}\right)=\frac{\operatorname{det}\left[A_{n, N_{f}-1,-N_{b}}\left(x_{N_{f}},\left\{x_{f}\right\}_{f \neq N_{f}} \mid\left\{y_{b}\right\}\right)\right]}{\prod_{j>i=1}^{N_{f}}\left(x_{j}^{2}-x_{i}^{2}\right) \prod_{i=1}^{N_{f}-1}\left(x_{N_{f}}^{2}-x_{i}^{2}\right)^{n-1}\left(2 x_{N_{f}}\right)^{n(n-1) / 2} \prod_{j>i=1}^{N_{b}}\left(y_{j}^{2}-y_{i}^{2}\right)},
$$

where we have introduced the $\left(N_{f}+n-1+N_{b}\right) \times\left(N_{f}+n-1+N_{b}\right)$ matrix

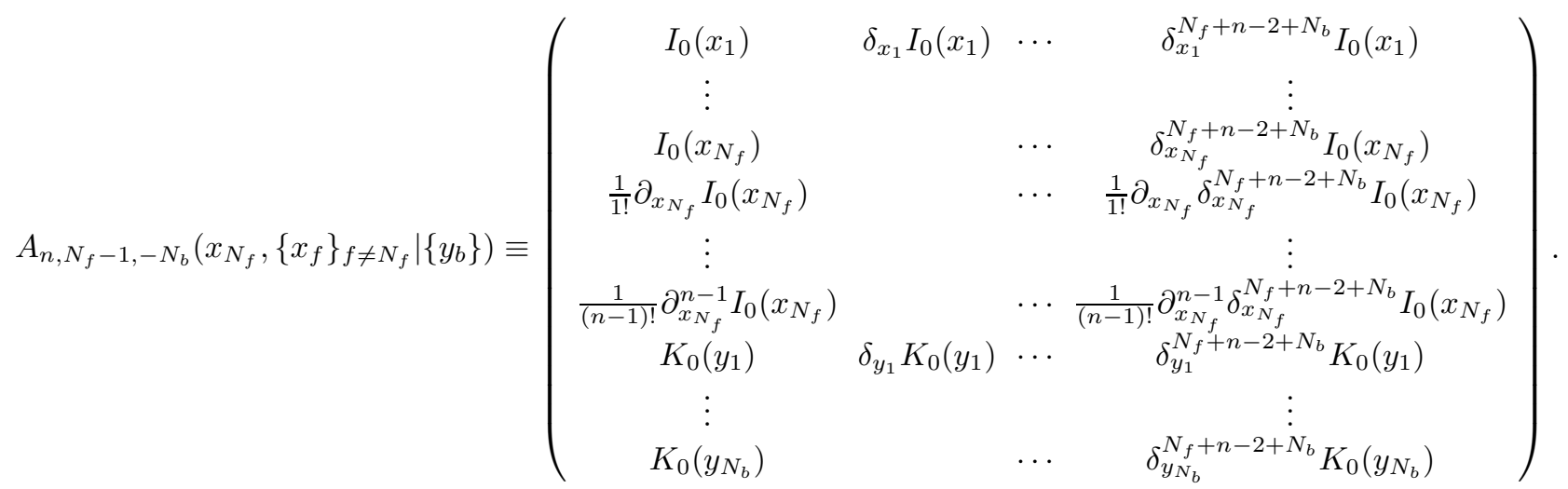

Using again the Sylvester identity, (45), this time with $i=N_{f}+n-1, j=N_{f}+n-1+N_{b}, p=N_{f}+n-2$, and $q=N_{f}+n-2+N_{b}$ we find the graded Toda lattice equation

$$
\begin{aligned}
\delta_{N_{f}+N_{b}} \delta_{x_{i}} \log Z_{n, N_{f}-1,-N_{b}}\left(x_{i},\left\{x_{f}\right\}_{f \neq i} \mid\left\{y_{b}\right\}\right) \\
=2 n x_{i}^{2} \frac{Z_{n+1, N_{f}-1,-N_{b}}\left(x_{i},\left\{x_{f}\right\}_{f \neq i} \mid\left\{y_{b}\right\}\right) Z_{n-1, N_{f}-1,-N_{b}}\left(x_{i},\left\{x_{f}\right\}_{f \neq i} \mid\left\{y_{b}\right\}\right)}{\left[Z_{n, N_{f}-1,-N_{b}}\left(x_{i},\left\{x_{k}\right\}_{f \neq i} \mid\left\{y_{b}\right\}\right)\right]^{2}} .
\end{aligned}
$$




\section{Appendix B. THE GRADED TODA LATTICE EQUATION WHERE TWO INDICES ARE AFFECTED}

In this appendix we consider the graded Toda lattice equations (19) and (20) relevant for the two-point functions. Although we have been able to check these equations in a number of cases including those relevant for quenching the Toda lattice equation we do not have a general proof.

The simplest possible example is

$$
\partial_{x} \partial_{y} \log Z_{1,-1}(x \mid y)=-4 x y \frac{Z_{2}(x) Z_{-2}(y)}{\left[Z_{1,-1}(x \mid y)\right]^{2}} .
$$

This result follows by rewriting the l.h.s. of this equation as

$$
\left[Z_{1,-1}(x \mid y)\right]^{2} \partial_{x} \partial_{y} \log Z_{1,-1}(x \mid y)=\operatorname{det}\left(\begin{array}{cc}
Z_{1,-1}(x \mid y) & \partial_{x} Z_{1,-1}(x \mid y) \\
\partial_{y} Z_{1,-1}(x \mid y) & \partial_{y} \partial_{x} Z_{1,-1}(x \mid y)
\end{array}\right)
$$

and using the identity

$$
\left(\begin{array}{cc}
Z_{1,-1}(x \mid y) & \partial_{x} Z_{1,-1}(x \mid y) \\
\partial_{y} Z_{1,-1}(x \mid y) & \partial_{y} \partial_{x} Z_{1,-1}(x \mid y)
\end{array}\right)=\frac{1}{2}\left(\begin{array}{cc}
Z_{1}(x) & \partial_{x} Z_{1}(x) \\
\partial_{x} Z_{1}(x) & \partial_{x}^{2} Z_{1}(x)
\end{array}\right)\left(\begin{array}{cc}
0 & 1 \\
1 & 0
\end{array}\right)\left(\begin{array}{cc}
Z_{-1}(y) & \partial_{y} Z_{-1}(y) \\
\partial_{y} Z_{-1}(y) & \partial_{y}^{2} Z_{-1}(y)
\end{array}\right)
$$

which valid if the partition function is of the form

$$
Z_{1,-1}(x \mid y)=\frac{1}{2}\left[Z_{1}(x) \delta_{y} Z_{-1}(y)+\delta_{x} Z_{1}(x) Z_{-1}(y)\right] .
$$

This structure of the supersymmetric partition function easily follows by integrating the bosonic variables before the Grassmann variables,

$$
\begin{aligned}
Z_{1,-1}(x \mid y) & =\int d \alpha d \beta I_{0}\left(x(1+\alpha \beta / 2) K_{0}(y(1-\alpha \beta / 2)\right. \\
& =\frac{1}{2}\left[I_{0}(x) \delta_{y} K_{0}(y)+\delta_{x} I_{0}(x) K_{0}(y)\right] .
\end{aligned}
$$

The general case: Our conjecture is that the generalization of the above graded Toda lattice equation is given by

$$
\delta_{x_{i}} \delta_{y_{j}} \log Z_{N_{f},-N_{b}}\left(\left\{x_{f}\right\} \mid\left\{y_{b}\right\}\right)=-4 n m x_{i}^{2} y_{j}^{2} \frac{Z_{N_{f}+1,-N_{b}+1}\left(x_{i},\left\{x_{f}\right\} \mid\left\{y_{b}\right\}_{b \neq j}\right) Z_{N_{f}-1,-N_{b}-1}\left(\left\{x_{f}\right\}_{f \neq i} \mid y_{j},\left\{y_{b}\right\}\right)}{\left[Z_{N_{f},-N_{b}}\left(\left\{x_{f}\right\} \mid\left\{y_{b}\right\}\right)\right]^{2}}
$$

where $n$ is the degeneracy of $x_{i}$ and $m$ is the degeneracy of $y_{j}$ in $Z_{N_{f},-N_{b}}\left(\left\{x_{f}\right\} \mid\left\{y_{b}\right\}\right)$, respectively. We have explicitly checked this equation for the cases listed in the table below:

\begin{tabular}{|c|c|c|c|c|} 
Eq $(61)$ & $N_{f}$ & $N_{b}$ & $n$ & $m$ \\
\hline case 1 & 1 & 1 & 1 & 1 \\
case 2 & 2 & 2 & 1 & 1 \\
case 3 & 2 & 2 & 2 & 1 \\
case 4 & 2 & 2 & 2 & 2 \\
case 5 & 3 & 1 & 3 & 1 \\
case 6 & 1 & 3 & 1 & 3
\end{tabular}
by

A similar equation holds when both derivatives are with respect to fermionic masses. It is conjectured to be given

$$
\delta_{x_{i}} \delta_{x_{j}} \log Z_{N_{f},-N_{b}}\left(\left\{x_{f}\right\} \mid\left\{y_{b}\right\}\right)=4 n m x_{i}^{2} x_{j}^{2} \frac{Z_{N_{f}+2,-N_{b}}\left(x_{i}, x_{j},\left\{x_{f}\right\} \mid\left\{y_{b}\right\}\right) Z_{N_{f}-2,-N_{b}}\left(\left\{x_{f}\right\}_{f \neq i, j} \mid\left\{y_{b}\right\}\right)}{\left[Z_{N_{f},-N_{b}}\left(\left\{x_{f}\right\} \mid\left\{y_{b}\right\}\right)\right]^{2}},
$$

where $n$ is the degeneracy of $x_{i}$ and $m$ is the degeneracy of $x_{j}$ in $Z_{N_{f},-N_{b}}\left(\left\{x_{f}\right\} \mid\left\{y_{b}\right\}\right)$, in this order. We have verified this identity for the following cases: 


\begin{tabular}{|c|c|c|c|c|} 
Eq $(62)$ & $N_{f}$ & $N_{b}$ & $n$ & $m$ \\
\hline case 1 & any & 0 & any & any \\
case 2 & 2 & 2 & 1 & 1 \\
case 3 & 3 & 1 & 2 & 1 \\
case 4 & 4 & 1 & 2 & 2 \\
case 5 & 4 & 1 & 3 & 1
\end{tabular}

[1] S.F. Edwards and P.W. Anderson, J. Phys. F5, 965 (1975).

[2] K.B. Efetov, Phys. Rev. Lett. 79, 491 (1997); Adv. Phys. 32, 53 (1983), Supersymmetry in disorder and chaos, (Cambridge University Press, Cambridge, 1997).

[3] M. Mézard, G. Parisi and M.A. Virasoro, Spin Glass Theory and Beyond, (World Scientific, Singapore 1987).

[4] J.J.M. Verbaarschot and M.R. Zirnbauer, Ann. Phys. 158, 78 (1984).

[5] F.J. Wegner, Z. Phys. 49, 297 (1983).

[6] P.H. Damgaard and K. Splittorff, Nucl. Phys. B 572, 478 (2000); Phys. Rev. D 62, 054509 (2000); P.H. Damgaard, Phys. Lett. B 476, 465 (2000).

[7] J.W. Negele and H. Orland, Quantum Many-Particle Systems, (Addison Wesley, 1998).

[8] R. Boels, J. de Boer, R. Duivenvoorden and J. Wijnhout, hep-th/0304061.

[9] H. Leutwyler, Phys. Rev. D 49, 3033 (1994).

[10] J. Gasser and H. Leutwyler, Ann. Phys. 158, 142 (1984); Nucl. Phys. B 250, 465 (1985); H. Leutwyler, Ann. Phys. 235, 165 (1994).

[11] J.J.M. Verbaarschot, H.A. Weidenmüller, and M.R. Zirnbauer, Phys. Rep. 129, 367 (1985).

[12] M.R. Zirnbauer, J. Math. Phys. 37, 4986 (1996).

[13] J.C. Osborn, D. Toublan and J.J.M. Verbaarschot, Nucl. Phys. B 540, 317 (1999).

[14] P.H. Damgaard, J.C. Osborn D. Toublan, and J.J.M. Verbaarschot, Nucl. Phys. B 547, 305 (1999).

[15] J.J.M. Verbaarschot and M.R. Zirnbauer, J. Phys. A 18, 1093 (1985).

[16] A. Kamenev and M. Mézard, J. Phys. A, 32, 4373 (1999); Phys. Rev. B 60, 3944 (1999).

[17] I.V. Yurkevich and I.V. Lerner, Phys. Rev. B 60, 3955 (1999).

[18] M.R. Zirnbauer, cond-mat/9903338.

[19] E. Kanzieper, Phys. Rev. Lett. 89, 250201 (2002).

[20] K. Splittorff and J.J.M. Verbaarschot, Phys. Rev. Lett. 90, 041601 (2003).

[21] K. Splittorff and J.J.M. Verbaarschot, accepted for publication in Nucl. Phys. B, hep-th/0310271.

[22] E. Kanzieper, cond-mat/0312006.

[23] M. Toda, Theory of Nonlinear Lattices, (Springer Series in Solid-State Sciences 20, Springer-Verlag, Second Edition 1989).

[24] Y.V. Fyodorov, Nucl. Phys. B 621, 643 (2002).

[25] S. Weinberg, Phys. Rev. 166, 1568 (1968).

[26] J. Gasser and H. Leutwyler, Phys. Lett. B 188, 477 (1987).

[27] H. Leutwyler and A. Smilga, Phys. Rev. D 46, 5607 (1992).

[28] E.V. Shuryak and J.J.M. Verbaarschot, Nucl. Phys. A 560, 306 (1993); J.J.M. Verbaarschot, Phys. Rev. Lett. 72, 2531 (1994).

[29] D. Dalmazi and J.J.M. Verbaarschot, Nucl. Phys. B 592, 419 (2001).

[30] R.C. Brower, P. Rossi and C-I. Tan, Nucl. Phys. B 190, 699 (1981); R.C. Brower and M. Nauenberg, Nucl. Phys. B 180, 221 (1981).

[31] J. B. Kogut, M. Snow and M. Stone, Nucl. Phys. B 200, 211 (1982).

[32] R. Dijkgraaf, H. Verlinde and E. Verlinde, Nucl. Phys. B 348, 435 (1991).

[33] A. Mironov, A. Morozov and G. Semenoff, Int. J. Mod. Phys. A 11, 5031 (1996).

[34] Y. V. Fyodorov and E. Strahov, Nucl. Phys. B 647, 581 (2002).

[35] Y.V. Fyodorov and E. Strahov, J. Phys. A 36, 3203 (2003).

[36] Y.V. Fyodorov and G. Akemann, JETP Lett. 77, 438 (2003).

[37] A.D. Jackson, M.K. Sener and J.J.M. Verbaarschot, Phys. Lett. B 387, 355 (1996).

[38] T. Guhr and T. Wettig, Nucl. Phys. B 506, 589 (1997).

[39] A.B. Balantekin, Phys. Rev. D 62, 085017 (2000).

[40] P. Forrester, Log-gases and Random matrices, Web Book - available at http://www.ms. unimelb. edu.au/ matpjf/matpjf.html. 
[41] K. Okamoto, Funkcialaj Ekvacioj 30, 305 (1987);

P.J. Forrester and N.S. Witte, Comm. Math. Phys. 219, 357 (2001);

P.J. Forrester and N.S. Witte, Comm. on Pure and Applied Math. 55, 679 (2002);

P.J. Forrester and N.S. Witte, math-ph/0204008;

M. Adler and P. van Moerbeke, Ann. of Math. 153, 149 (2001); math.CO/9912143;

P. van Moerbeke, math/0010135.

[42] S. Kharchev, A. Marshakov, A. Mironov, A. Morozov and A. Zabrodin, Nucl. Phys. B 380, 181 (1992).

[43] D. Toublan and J.J.M. Verbaarschot, Nucl. Phys. B 603, 343 (2001).

[44] J.J. Sylvester, Compt. Rend. Acad. Sc. 54, 129 (1862). 